\title{
Exceeding the Nonlinear-Shannon Limit using Raman Laser Based Amplification and Optical Phase Conjugation
}

\author{
I.D. Phillips, M. Tan , M.F.C. Stephens, M. E. McCarthy, E. Giacoumidis, S. Sygletos, P. Rosa, S. Fabbri, \\ S. T. Le, T. Kanesan, S. K. Turitsyn, N. J. Doran, P. Harper, A. D. Ellis \\ Aston Institute of Photonic Technologies, Aston University, Birmingham, B4 7ET, UK \\ i.phillips@aston.ac.uk
}

\begin{abstract}
We demonstrate that a combination of Raman laser based amplification and optical phase conjugation enables transmission beyond the nonlinear-Shannon limit. We show nonlinear compensation of 7x114Gbit/s DP-QPSK channels, increasing system reach by 30\%.

OCIS codes: (060.2320) Fiber optics amplifiers and oscillators; (070.5040) Phase conjugation
\end{abstract}

\section{Introduction}

The so called nonlinear-Shannon limit is now well understood [1] and the consequences of this limit are leading to proposals to combat inter-channel nonlinear effects using digital back propagation (DBP) [2], transmitter based phase conjugation [3], and mid-span phase conjugation [4]. The complexity of DBP has restricted performance gains to around $1 \mathrm{~dB}$ [2], and recent demonstrations of optical phase conjugation have produced marginal net benefits [4]. Distributed Raman amplification has been shown to not only enhances OSNR, but can also be used to substantially reduce the effect of fiber nonlinearities [5].

In this paper, we demonstrate that polarization insensitive optical phase conjugation (OPC) may indeed be used to significantly reduce the impact of nonlinear inter-channel interactions. For efficient nonlinearity compensation, OPC relies on a symmetric signal power profile [6] which is enabled in this paper by the use of second-order ultralong Raman fiber laser (URFL) based amplification, which simultaneously enhances the optical signal to noise ratio of the link. In particular, we show that the reach of 7x114Gbit/s DP-QPSK channels is increased from 5,200km (EDFA only) to more than 10,400km (Raman plus OPC) resulting from a 50\% increase in reach due to the OSNR benefits of Raman amplification and a further 70\% reduction in the impact of nonlinear impairments due to OPC. These results are within 25\% of the limit imposed by parametric noise amplification [7] and exceed the conventional nonlinear-Shannon limit for lossless amplification for this system (including the loop specific losses).

\section{Experimental Setup}

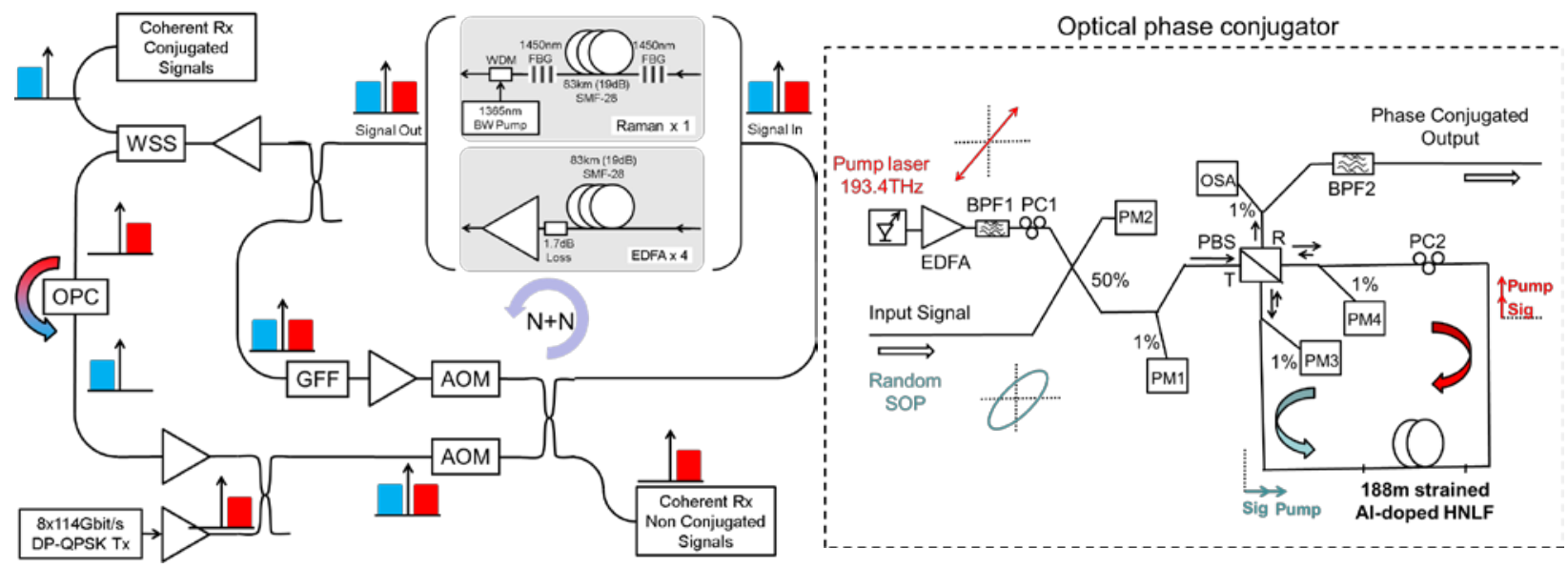

Figure 1. Experimental configuration of (a) the dual input recirculating loop and (b) optical phase conjugator.

The experimental setup in shown in Figure 1 which consists of an 8x114Gbit/s dual polarization quadrature phase shift keying (DP-QPSK) transmitter, a recirculating loop, an optical phase conjugator and a coherent receiver, each of which is described in more detail below.

The transmitter consisted of 8 distributed feedback (DFB) lasers at 100GHz spacing from 1551.72nm (193.2THz) to $1557.36 \mathrm{~nm}(192.5 \mathrm{THz})$ which were multiplexed using an arrayed waveguide grating. The output was combined with a narrow linewidth $(100 \mathrm{kHz})$ external cavity laser, which replaced each DFB during the measurement of that channel. The continuous-wave (CW) channels were modulated using a Mach-Zehnder I/Q 
modulator driven by 28.5Gbit/s, $2^{31}-1$ word length, normal and inverted PRBS patterns (18bit relative delay) from a pulse pattern generator, thus generating 8x28.5Gbaud QPSK signals. The output was then passively polarization multiplexed (2ns relative delay) to form 8x114Gbit/s DP-QPSK signals.

To allow a single recirculating loop to be used for transmission of both original signal channels in the 1550$1560 \mathrm{~nm}$ range and conjugated channels in the 1540-1550nm range, a dual input configuration was used on the loop input path. To load the loop, the original signal channels were combined with their conjugates (after transmission round the loop), such that both conjugated and non-conjugated channel blocks were simultaneously present at the loop input for loading, and the OPC continuously conjugates the signal channels. During transmission, the original signal channels and their conjugates were transmitted on the inner loop for $\mathrm{N}$ circulations. To assess the performance without OPC the original channels were coherently detected after exiting the loop at the main loop coupler (bottom right). A portion of the original channels would also have passed through the OPC for phase conjugation and the AOM's were timed such that the conjugates were re-coupled into the loop along with newly generated signals from the main transmitter, transmitted a further $\mathrm{N}$ circulations and then measured (after a total of $2 \mathrm{~N}$ circulations) at the output of the WSS (top left) used for band selection at the OPC input. A gain flattering filter was used to equalize the channels during each recirculation.

We used a $2^{\text {nd }}$ order backward pumped URFL amplification technique, which has been shown to give quasilossless transmission conditions over a wide wavelength range [6]. This provides a symmetric power profile which is required for nonlinear compensation, and therefore has the potential to offer superior transmission performance over both conventional EDFA based system and $1^{\text {st }}$ order Raman based systems. This consisted of a single $83 \mathrm{~km}$ span of SMF-28 ( 19dB loss) surrounded by a matched pair of fiber Bragg gratings (FBG), see Figure 1. The FBG pair has a matched center wavelength in the $1448 \mathrm{~nm}$ range, a $0.5 \mathrm{~nm} 3 \mathrm{~dB}$ bandwidth a $95 \%$ reflectivity and $1.7 \mathrm{~dB}$ insertion loss (including loss from WDM couplers). A backwards propagating $1365 \mathrm{~nm}$ pump was used to induce lasing in the $83 \mathrm{~km}$ long fiber, and the resultant bi-directionally propagating $1448 \mathrm{~nm}$ Raman fiber laser pumped the C-band communication signals. The signal power distribution along the span was measured with a modified optical time-domain reflectometry (OTDR) technique [6] revealing an almost symmetric power distribution with a maximum peak to peak signal excursion of only $6 \mathrm{~dB}$ (see inset to Figure 2a).

As a bench mark comparison, a four span ( 83km, 19dB loss) conventional gain flattened EDFA (5.5dB noise figure) based system was also characterized. To emulate a practical system the loss budget of this system included additional insertion losses at the input and output of each span, and (unlike the Raman system) the impact of loop specific losses were minimized by the use of four spans.

Optical phase conjugation was achieved using four-wave mixing in a polarization diverse scheme as shown in Figure $1 \mathrm{~b}$. The incoming data signals $(\sim 5 \mathrm{dBm} /$ channel) were combined with a high power $(\sim 37 \mathrm{dBm})$ pump signal at 193.4THz. The pump and signal were injected bi-directionally using a $2 \times 2$ polarization beam splitter (PBS) into $188 \mathrm{~m}$ of highly nonlinear fiber (HNLF) which was configured in a loop (loss, nonlinear coefficients and SBS threshold of $2.6 \mathrm{~dB}, 6.9(\mathrm{~W} . \mathrm{km})^{-1}$ and $30 \mathrm{dBm}$ respectively). The pump polarization was controlled to be at a $45^{\circ}$ linear polarization, such that equal powers propagated in each direction around the loop, in orthogonal polarizations. The counter-propagating orthogonal pump signals interacted with the component of signal in the same polarization to produce two sets of orthogonal conjugates which were polarization re-combined through the same PBS. The conjugates were filtered and amplified before re-transmission. Total input signal to conjugate loss for the OPC is $\sim 20 \mathrm{~dB}$ including $\sim 5 \mathrm{~dB}$ conversion efficiency from signal to conjugate.

At the coherent receiver, the signal was fed through a $100 \mathrm{GHz}$ tunable filter followed by an EDFA, before being combined with a narrow linewidth $(\sim 100 \mathrm{kHz})$ local oscillator in a polarization diverse 90-degree optical hybrid. The outputs from the optical hybrid were detected using balanced receivers, recovering the in-phase (I) and quadrature $(\mathrm{Q})$ signals in the $\mathrm{X}$ - and $\mathrm{Y}$ - polarizations. The four signals were sampled and digitized using an 80Gs/s real-time oscilloscope with a $36 \mathrm{GHz}$ analog bandwidth. The data was processed using off-line DSP including signal normalization, low pass filtering, down sampling, static dispersion compensation, clock recovery, polarization recovery (constant modulus algorithm) and phase recovery. Q-factors were estimated from the error vector magnitude, and averaged over 10 sets of $57 \mathrm{kSymbols}$. All results were fitted to a nonlinear-Shannon model based on four wave mixing [8] including additional noise terms associated with the transmitter OSNR, loop and OPC insertion losses and parametric noise amplification.

\section{Results and Discussion}

Figure 2a shows baseline results using EDFA amplification with and without phase conjugation. Without OPC, excellent agreement with both nonlinear threshold measurements (top right inset) and the length dependence (main figure) was observed once loop specific losses of $12 \mathrm{~dB}$ were taken into account. For OPC results, the efficiency of non-linearity compensation was used as a fitting parameter. This reveals that the observed $2 \mathrm{~dB}$ increase in nonlinear 
threshold was associated with $40 \%$ compensation of nonlinear impairments by the OPC. A marginal improvement in the maximum 5,200km transmission distance was observed, due to the additional penalty associated with the OPC insertion loss, as observed by other authors. This poor performance is primarily due to the lack of power symmetry in the transmission link. The bottom left shows $\mathrm{Q}$ factor vs. frequency for all channels at the maximum transmission distance with and without OPC.
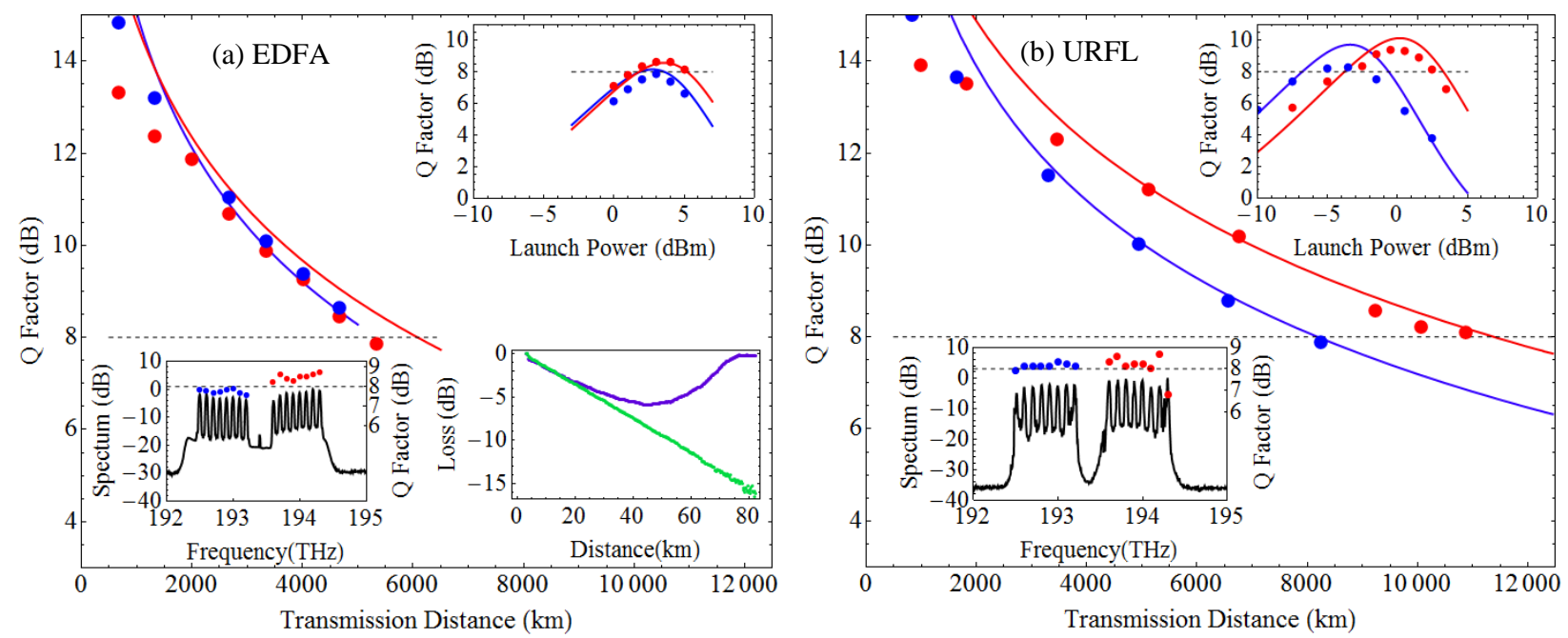

Figure 2. (a) EDFA transmission; (b) URFL amplified transmission. Main figures: $\mathrm{Q}^{2}$ vs. transmission distance. Insets: $\mathrm{Q}^{2}$ vs. Launch Power; Spectra and $\mathrm{Q}^{2}$ vs. Frequency at $\mathrm{Q}^{2}=8 \mathrm{~dB}$; Loss vs. distance for EDFA (green) and QLRA (blue); Blue circles: experimental non-OPC

channels; Red circles: experimental OPC channels; Blue line: theoretical non-OPC channels; Red line: theoretical OPC channels

Figure $2 \mathrm{~b}$ shows equivalent results for URFL amplification, which significantly outperforms the phase conjugated EDFA system, thanks to the improved noise performance with at least a $50 \%$ increase in reach. Furthermore, due to the enhanced power symmetry, the nonlinear compensation is greatly enhanced, allowing an increase in nonlinear threshold of around $3 \mathrm{~dB}$ (70\% compensation of nonlinearity) and a significant net increase in transmission reach of more than $30 \%$ with the inclusion of OPC, and a total doubling of the transmission reach compared to the EDFA system, even with the enhanced loop specific noise degradations. We believe that of the remaining 30\% of the nonlinear penalty, $\sim 9 \%$ arises from the incomplete phase conjugation (only 8 out of 16 channels are conjugated) and the remaining $21 \%$ due to the residual power asymmetry and the effects of PMD.

\section{Conclusions}

We have experimentally demonstrated that a novel combination of Raman laser based amplification and optical phase conjugation allows the transmission distance of 7x114Gbit/s DP-QPSK signals (104Gbit/s pre-FEC) to be extended by $3 \mathrm{~dB}$ from $5200 \mathrm{~km}$ (EDFA system) to $10400 \mathrm{~km}$. This system compensates for $70 \%$ of the nonlinear impairments in a dispersion uncompensated single mode fiber system, which represents a significant improvement over reported DSP based nonlinearity compensation.

\section{Acknowledgements}

This work was partly funded by the EPSRC funded projects UNLOC (EP/J017582/1) and FOPA (EP/J009709/1), European Community projects FOX-C (318415) and DISCUS (318137); and The Royal Society (WM120035TEST). The authors would like to thank Prof. Lin Zhang and Sun Zhongyuan for the FBG's used in this experiment.

\section{References}

[1] P.Poggiolini, "Modeling of Non-Linear Propagation in Uncompensated Coherent Systems”, OFC 2013, OTh3G1

[2] D.Levery et al, “Long-Haul Transmission of PS-QPSK at $100 \mathrm{~Gb} / \mathrm{s}$ Using Digital Backpropagation”, PTL 24(3), pp176, (2012)

[3] M. D. Pelusi et al, "Optically tunable compensation of nonlinear signal distortion in optical fiber by end-span optical phase conjugation," Opt. Express 20, pp8015 (2012)

[4] L. B. Du, et al, "Fiber nonlinearity compensation for OFDM super-channels using optical phase conjugation," Opt. Expr 20, pp19921 (2012)

[5] A. Carena, et al. "On the optimization of hybrid Raman/erbium-doped fiber amplifiers." PTL 13(11), pp1170-1172, (2001)

[6] J.D. Ania-Castañón, et al, "Simultaneous spatial and spectral transparency in ultra-long fiber lasers", Phys. Rev. Lett. 101 (2008), 123903

[7] D. Rafique and A. D. Ellis, "Impact of signal-ASE four-wave mixing on the effectiveness of digital back-propagation in 112 Gb/s PM-QPSK systems”, Optics Express, Vol. 19, No. 4, pp3449, (2011)

[8] A.D.Ellis, “The MODE-GAP project”, IEEE Photonics Conference (IPC), Paper 299445, (2013). 